IP $\rightleftharpoons$ B

\title{
O componente arbustivo-arbóreo em área de Depressão Sertaneja Meridional em Petrolina, PE
}

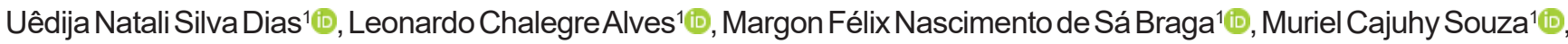 \\ Luiz Felipe Flor da Silva ${ }^{1}$, William de Souza e Sousa ${ }^{1}$ (iD), Fabrício Alvim Carvalho ${ }^{2}$, Daniel Salgado Pifano ${ }^{1 *}$ (iD)
}

${ }^{1}$ Universidade Federal do Vale do São Francisco, Rod. BR 407, Km 12, Lote 543, s/nº - C1, CEP 56300-990, Petrolina, PE, Brasil

${ }^{2}$ Universidade Federal de Juiz de Fora, Rua José Lourenço Kelmer, S/n, Martelos, CEP 36036-330 Juiz de Fora, MG, Brasil

"Autor correspondente:

daniel.pifano@univasf.edu.br

Termos para indexação:

Semiárido

Fitossociologia

Endemismo

\section{Index terms:}

Semiarid

Phytossociology

Endemism

Histórico do artigo:

Recebido em 21/09/2017

Aprovado em 09/07/2018

Publicado em 11/12/2018

doi: 10.4336/2018.pfb.38e201701502
Resumo - Objetivou-se avaliar a estrutura, diversidade e a heterogeneidade do componente arbustivo-arbóreo de Caatinga da Depressão Sertaneja Meridional em Petrolina, PE. Foram alocadas 25 parcelas de $400 \mathrm{~m}^{2}$, sendo medidas a altura total e a circunferência ao nível do solo (CAS $\geq 15,8 \mathrm{~cm}$ ) dos indivíduos vivos ou mortos em pé, para o cálculo dos parâmetros fitossociológicos. A heterogeneidade da comunidade foi avaliada pela análise de correspondência destendenciada. Foram mensurados 825 indivíduos, distribuídos em 11 famílias, 22 gêneros e 27 espécies. As famílias com maior riqueza foram Fabaceae (9 spp.) e Euphorbiaceae (5 spp.), totalizando 51,8\% da riqueza total. As espécies com maiores valores de cobertura e de importância foram Mimosa tenuiflora e Poincianella microphylla, justificado por suas elevadas abundâncias (295 e 217 indivíduos, respectivamente) e áreas basais $\left(2,95\right.$ e 1,72 $\mathrm{m}^{2} \mathrm{ha}^{-1}$, respectivamente). Foram encontrados valores considerados medianos para os índices de diversidade $\left(H^{\prime}=2,04\right)$ e equabilidade $\left(J^{\prime}=0,62\right)$, e houve ajuste ao modelo Log normal de distribuição de espécies, sugerindo dominância ecológica moderada das espécies mencionadas. A comunidade estudada se mostrou bastante heterogênea, possivelmente em função do uso múltiplo do solo no passado, reforçando a responsabilidade de instituições com remanescentes florestais de estabelecerem medidas para a conservação de suas áreas verdes.

\section{The woody component in a Southern Sertanean Depression area, in Petrolina, Pernambuco State, Brazil}

\begin{abstract}
The aim of this study was to evaluate the structure and diversity of the woody component of Caatinga in Southern Sertanean Depression, Petrolina, Pernambuco State, Brazil. We sampled 25 plots of $400 \mathrm{~m}^{2}$. Total height and circumference at the soil level $(\mathrm{CSL} \geq 15.8 \mathrm{~cm})$ were measured of living or dead standing individuals, to calculate phytossociological parameters. The community heterogeneity was evaluated through detrended correspondence analyzes. We sampled 825 individuals, distributed in 11 families, 22 genera and 27 species. The families with the highest richness were Fabaceae (9 spp.) and Euphorbiaceae (5 spp.), with 51.8\% of the total richness. The species with higher cover value and importance value were Mimosa tenuiflora and Poincianella microphylla, justified by their high abundances (295 and 217 individuals, respectively) and basal areas (2.95 and $1.72 \mathrm{~m}^{2} \mathrm{ha}^{-1}$, respectively). We found median values for the diversity and equability indices $\left(\mathrm{H}^{\prime}=2.04\right.$ and $\left.\mathrm{J}^{\prime}=0.62\right)$, and species distribution presented better adjust to Log normal model, suggesting moderate ecological dominance of these species. The studied community was very heterogeneous, possibly due to the multiple use of the soil in the past, reinforcing the responsibility of institutions with forest remnants to establish measures for the conservation of their green areas.
\end{abstract}




\section{Introdução}

A região nordeste brasileira estende-se por uma área de $1.548 .672 \mathrm{~km}^{2}$. A Caatinga é a vegetação que recobre $844.453 \mathrm{~km}^{2}$ dessa área, equivalendo a cerca de $86 \%$ do semiárido. Encontra-se distribuída por todos os Estados do Nordeste e parte do norte de Minas Gerais. Em extensão territorial é o terceiro maior bioma brasileiro, distribuindo-se em $54 \%$ da região Nordeste e $11 \%$ do território nacional (Alves, 2007). É um bioma exclusivamente brasileiro, rico em espécies nativas e endêmicas, embora seja o menos conhecido pela ciência e o menos protegido por unidades de conservação (Brasil, 2002).

A Caatinga apresenta características bem definidas, como a grande variação fisionômica, principalmente quanto ao porte e densidade da vegetação, onde são encontrados arbustos e árvores que geralmente perdem as folhas na estação seca (Alves et al., 2009). Segundo Rodal et al. (2013), a diversificação na composição e na fisionomia da vegetação, retratada principalmente na estratificação horizontal e vertical das comunidades, está condicionada a fatores abióticos, como escassez hídrica, profundidade do solo, descontinuidades litológicas nos perfis, salinidade, relevo e a constituição mineralógica das formações superficiais.

Considerando toda esta heterogeneidade associada ao crescimento econômico e demográfico da região nordeste, tem-se um cenário de profunda degradação da Caatinga, aumentando a área em condição de desertificação em muitas ecorregiões, principalmente naquelas com maior índice de aridez, como a depressão sertaneja meridional (DSM) (Centro de Gestão e Estudos Estratégicos, 2016). Assim, torna-se prioritário que sejam reconhecidas quais espécies vegetais nativas são capazes de resistir à perturbação antrópica, para compor o conhecimento necessário aos trabalhos de recuperação da Caatinga. Diante disso, destaca-se a necessidade de se conhecer a flora do bioma, por levantamentos de vegetação, tanto qualitativos como quantitativos.

Este cenário motivou a elaboração do presente estudo, já que a grande maioria dos remanescentes de Caatinga na Depressão Sertaneja encontram-se com severo histórico de uso antrópico, principalmente na região do Vale do São Francisco. Em adição, tal região constitui o maior polo urbano do sertão (mais de 600 mil habitantes) com forte atividade agrícola, fruto do sistema de irrigação suportado pelo Rio São Francisco (Ortega \& Sobel, 2010). Tal atividade exerce forte pressão sobre remanescentes de caatinga na região e os poucos restantes não possuem qualquer ação voltada à sua conservação.

Portanto, objetivou-se avaliar a estrutura, a diversidade e a heterogeneidade do componente arbustivo-arbóreo em área de Caatinga pertencente à maior ecorregião do bioma, a depressão sertaneja meridional, em Petrolina, $\mathrm{PE}$, reconhecendo as espécies endêmicas, raras e ameaçadas de extinção.

\section{Material e métodos}

\section{Área de estudo}

O trabalho foi realizado no Campus de Ciências Agrárias (CCA) da Universidade Federal do Vale do São Francisco (UNIVASF), localizado no município de Petrolina, sertão pernambucano. O CCA encontra-se inserido geologicamente na Província do Sub-Médio São Francisco, unidade geoambiental ou ecorregião da Depressão Sertaneja Meridional (DSM) (Beltrão et al., 2005). A sede do campus situa-se a uma latitude 09¹9'29' S, longitude 40 32'59' $\mathrm{W}$, e altitude de $389 \mathrm{~m}$ (Beltrão et al., 2005).

O CCA corresponde a uma área com solo predominantemente arenoso, classificado como Argissolo ou Podzólico Amarelo, típico das adjacências do rio São Francisco (Araújo Filho et al., 2000), com históricos de uso e ocupação heterogêneos. O clima da região é do tipo Semiárido (BSh segundo a classificação de Köppen), com chuvas de verão e precipitação média anual de 693,0 mm (Brasil, 2006).

Antes pertencente à Codevasf, no local havia áreas de cultivo e sequeiro (vegetação savânica sem atividade antrópica), onde na primeira houve desmatamento total (corte raso) e na segunda apenas corte seletivo (Souza et al., 2013). Como pode ser visto na Figura 1, há visível diferença entre as áreas, sendo as de plantio correspondentes às parcelas $01,02,03,04,06,09,10 \mathrm{e}$ 11 e as de sequeiro as demais parcelas. O CCA continua em processo de construção, o que tende a aumentar a fragmentação do remanescente vegetacional nativo, que hoje é de cerca de 50 ha. A vegetação é composta por Savana estépica arborizada, com trechos de Floresta Caducifólia (IBGE, 2012). 


\section{Coleta de dados e amostragem}

Para verificar os padrões estruturais do componente arbustivo-arbóreo, foi utilizado o método de parcelas (Martins, 1991), onde foram alocadas 25 parcelas de $400 \mathrm{~m}^{2}(20 \mathrm{~m} \times 20 \mathrm{~m})$, totalizando 1 hectare amostrado. A alocação das mesmas foi realizada em campo conforme extensão e formato da vegetação, todas foram georreferenciadas (Figura 1).

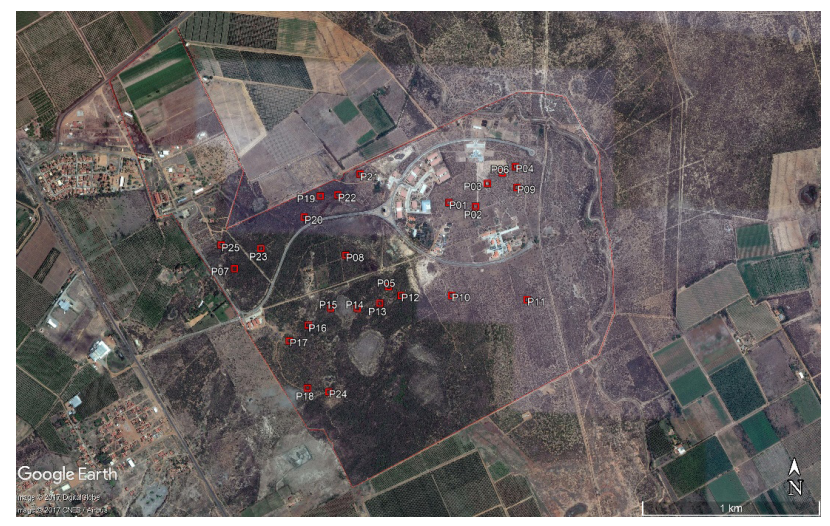

Figura 1. Distribuição das parcelas na área de estudo, o Campus de Ciências Agrárias da UNIVASF. Fonte: Google Earth (C) 2017 Europa Tecnologie - Image (C) 2017 DigitalGlobe.

Figure 1. Distribution of the plots in the study area, UNIVASF Campus of Agricultural Sciences. Source: Google Earth (C) 2017 Europa Tecnologie - Image () 2017 DigitalGlobe.

Foram contemplados na amostragem apenas indivíduos eretos e lenhosos, com circunferência à altura do solo $(\mathrm{CAS}) \geq 15,8 \mathrm{~cm}$, incluindo as suculentas (Cactaceae). Todos os indivíduos amostrados foram marcados com plaquetas de alumínio para atender às exigências para estudos futuros de dinâmica. As alturas foram estimadas em campo com o auxílio da vara extensora de $2 \mathrm{~m}$ do podão, realizada sempre pelo mesmo membro da equipe. As medidas de circunferência foram tomadas com auxílio de fita métrica sem uso anterior (nova) de $1,5 \mathrm{~m}$ de comprimento. Os indivíduos mortos em pé (dentro do critério) também foram amostrados (categoria $=$ morto).

Para testar a suficiência amostral, foi realizado o cálculo dos estimadores de riqueza CHAO 1 e CHAO 2, com o programa EstimateS, partindo do pressuposto que a sobreposição entre os resultados observado e esperado para os dois estimadores num intervalo de confiança de 95\% indicam uma amostragem suficiente (Schilling \& Batista, 2008; Colwell et al., 2012).
As espécies foram identificadas em campo e confirmadas em laboratório, utilizando-se chaves de identificação e/ou pela comparação com as exsicatas depositadas na coleção do Núcleo de Ecologia e Monitoramento Ambiental (NEMA) da UNIVASF. Os materiais encontrados em estágio fenológico reprodutivo foram poucos e eram espécies que já haviam sido coletadas e incorporadas à coleção em oportunidades anteriores. A classificação das famílias foi realizada segundo a APG IV (The Angiosperm Phylogeny Group, 2016), com o nome dos autores organizados segundo Brummitt \& Powell (1992) e as sinonímias e endemismos checados através do site Flora do Brasil (2017). A raridade na amostragem foi definida segundo Martins (1991) e o status conservacionista de cada espécie foi verificado na lista vermelha da flora do Brasil (Martinelli \& Moraes, 2013).

\section{Análises}

Para descrição da estrutura da comunidade arbustivoarbórea, foram calculados os seguintes parâmetros fitossociológicos (Mueller-Dombois \& Ellenberg, 1974): área basal $\left(\mathrm{m}^{2}\right)$, densidades absoluta e relativa, frequências absoluta e relativa, dominâncias absoluta e relativa e valores de importância e de cobertura. Histogramas de classes de diâmetro e altura foram construídos a partir dos atributos pertinentes, com intervalos crescentes entre as classes (Rodal et al., 2013).

Para a diversidade de espécies, foram calculados os índices de Shannon (H') e de equabilidade de Pielou (J') (Begon et al., 1996). Complementarmente, testou-se o ajuste da comunidade em três modelos de distribuição de espécies: geométrico, de vara-quebrada e normal-logarítmico, utilizando-se o logaritmo na base 10 (Magurran, 1988). A significância da distribuição das abundâncias em cada um dos três modelos foi testada através de qui-quadrado $\left(\chi^{2}\right)$, considerando que um valor abaixo do nível de probabilidade de $95 \%$ $(\mathrm{P}<0,05)$ indica a ausência de ajuste ao modelo (Hammer et al., 2008).

A heterogeneidade das espécies na comunidade foi analisada por correspondência destendenciada (DCA). A partir dessa análise, tem-se, indiretamente, o produto da variabilidade na distribuição das espécies pelas parcelas ao longo da comunidade (Felfili et al., 2005). Para a realização da DCA foi construída uma matriz quantitativa de abundância das espécies por parcela. Os dados de espécies não sofreram padronização ou transformação, uma vez que a riqueza foi baixa 
e distribuída, em sua maioria, também em baixas densidades. As análises foram realizadas no programa PAST (Hammer et al., 2008).

\section{Resultados}

Foram registrados 825 indivíduos distribuídos em 11 famílias, 22 gêneros e 27 espécies (Tabela 1). As famílias Fabaceae e Euphorbiaceae foram as mais representativas. Fabaceae esteve presente com 9 espécies $(33,3 \%)$ e Euphorbiaceae com 5 (18,5\%), totalizando $51,8 \%$ da riqueza total.

Os gêneros mais frequentes foram Pilosocereus, Erythroxylum, Croton, Mimosa, Poincianella e Pseudobombax, cada um deles representado por duas espécies. Mimosa tenuiflora e Poincianella microphylla foram as espécies mais abundantes e frequentes na amostra, sendo registradas em $84 \%$ das parcelas existentes. M. tenuiflora e P. microphylla foram representadas por 295 e 217 indivíduos, respondendo por $35,76 \%$ e $26,3 \%$ da densidade relativa total, respectivamente. Combretum monetaria, Cynophalla hastata, Erythroxylum pugens, Pilosocereus pachycladus e Senna macranthera var. pudibunda, foram consideradas raras na amostragem (sensu Martins, 1991), com o registro de apenas um indivíduo cada. Estando presentes em apenas 4\% das unidades amostrais, as mesmas apresentaram reduzidas áreas basais (AB) $\left(0,003 ; 0,009 ; 0,004 ; 0,013\right.$ e $0,004 \mathrm{~m}^{2} \mathrm{ha}^{-1}$, respectivamente), mostrando serem indivíduos de pequeno porte e com distribuição restrita.

Entre as espécies encontradas, 40,7\% são endêmicas da Caatinga, tais como: Pilosocereus gounellei, Pilosocereus pachycladus, Cnidoscolus quercifolius, Combretum monetaria, Croton blanchetianus, Erythroxylum revolutum, E. pugens, Pityrocarpa moniliformis, Poincianella microphylla, P. pyramidalis, Pseudobombax simplicifolium e Spondias tuberosa (Flora do Brasil, 2017).

O valor de área basal $(\mathrm{AB})$ para a comunidade foi de $8,81 \mathrm{~m}^{2}$ ha $^{-1}$, com destaque para umburana-de-cambão (Commiphora leptophloeos), com terceira maior $\mathrm{AB}$ $\left(1,02 \mathrm{~m}^{2} \mathrm{ha}^{-1}\right)$ e densidade absoluta (72 ind ha-1). O índice de diversidade Shannon-Weaner encontrado foi de $\mathrm{H}^{\prime}=2,04$ nat $\operatorname{sind}^{-1}$ e o valor de equabilidade foi J' $=0,62$, denotando moderada dominância ecológica das duas espécies mais importantes na comunidade sobre as demais.

Os estimadores de riqueza CHAO 1 e CHAO 2 (Tabela 2) mostraram que a suficiência amostral foi estatisticamente atingida, já que houve sobreposição entre o observado e o esperado para os dois estimadores num intervalo de confiança de 95\% (Colwell et al., 2012).

Tabela 1. Parâmetros fitossociológicos das espécies amostradas em remanescente na Depressão Sertaneja Meridional, Petrolina, PE.

Table 1. Phytosociological parameters of the species sampled in remnant in Southern Sertaneja Depression, Petrolina, PE

\begin{tabular}{|c|c|c|c|c|c|c|c|c|c|c|}
\hline Família & Espécies & $\begin{array}{l}\mathbf{A B} \\
\left(\mathbf{m}^{2}\right)\end{array}$ & $\begin{array}{c}\text { DA } \\
\text { (ind ha-1) }\end{array}$ & $\begin{array}{l}\text { DR } \\
(\%)\end{array}$ & $\begin{array}{l}\text { FA } \\
(\%)\end{array}$ & $\begin{array}{l}\text { FR } \\
(\%)\end{array}$ & $\begin{array}{c}\text { DoA } \\
\left(\mathrm{m}^{2} \mathbf{h a}^{-1}\right)\end{array}$ & $\begin{array}{l}\text { DoR } \\
(\%)\end{array}$ & $\mathbf{V C}$ & VI \\
\hline \multirow[t]{2}{*}{ Fabaceae } & Mimosa tenuiflora (Willd.) Poir. & 2,954 & 295 & 35,76 & 0,84 & 15,00 & 871,52 & 63,23 & 98,99 & 113,99 \\
\hline & $\begin{array}{l}\text { Poincianella microphylla (Mart. Ex } \\
\text { G. Don) L. P. Queiroz }\end{array}$ & 1,728 & 217 & 26,30 & 0,84 & 15,00 & 374,97 & 27,20 & 53,51 & 68,51 \\
\hline Burseraceae & $\begin{array}{c}\text { Commiphora leptophloeos (Mart.) J. } \\
\text { B. Gillett }\end{array}$ & 1,027 & 72 & 8,73 & 0,52 & 9,29 & 73,98 & 5,37 & 14,09 & 23,38 \\
\hline Fabaceae & Piptadenia stipulacea (Benth.) Ducke & 0,330 & 45 & 5,45 & 0,4 & 7,14 & 14,86 & 1,08 & 6,53 & 13,68 \\
\hline Euphorbiaceae & Cnidoscolus quercifolius Pohl & 0,635 & 29 & 3,52 & 0,52 & 9,29 & 18,41 & 1,34 & 4,85 & 14,14 \\
\hline Fabaceae & $\begin{array}{c}\text { Poincianella pyramidalis (Tull.) L. } \\
\text { P. Queiroz }\end{array}$ & 0,188 & 31 & 3,76 & 0,08 & 1,43 & 5,83 & 0,42 & 4,18 & 5,61 \\
\hline Euphorbiaceae & Croton rhamnifolius Willd. & 0,134 & 29 & 3,52 & 0,28 & 5,00 & 3,88 & 0,28 & 3,80 & 8,80 \\
\hline Fabaceae & Mimosa hexandra Micheli & 0,193 & 27 & 3,27 & 0,08 & 1,43 & 5,20 & 0,38 & 3,65 & 5,08 \\
\hline Apocynaceae & Aspidosperma pyrifolium Mart. & 0,218 & 14 & 1,70 & 0,2 & 3,57 & 3,05 & 0,22 & 1,92 & 5,49 \\
\hline Fabaceae & $\begin{array}{l}\text { Pityrocarpa moniliformis (Benth.) } \\
\text { Luckow \& R. W. Jobson }\end{array}$ & 0,152 & 11 & 1,33 & 0,12 & 2,14 & 1,68 & 0,12 & 1,45 & 3,60 \\
\hline Malvaceae & $\begin{array}{c}\text { Pseudobombax simplicifolium A. } \\
\text { Robins }\end{array}$ & 0,353 & 7 & 0,85 & 0,16 & 2,86 & 2,47 & 0,18 & 1,03 & 3,88 \\
\hline
\end{tabular}


Tabela 1. Continuação.

Table 1. Continuation.

\begin{tabular}{|c|c|c|c|c|c|c|c|c|c|c|}
\hline Família & Espécies & $\begin{array}{l}\mathrm{AB} \\
\left(\mathbf{m}^{2}\right)\end{array}$ & $\begin{array}{c}\text { DA } \\
\text { (ind hat } \\
\end{array}$ & $\begin{array}{l}\text { DR } \\
(\%)\end{array}$ & $\begin{array}{c}\text { FA } \\
(\%)\end{array}$ & $\begin{array}{l}\text { FR } \\
(\%)\end{array}$ & $\begin{array}{c}\text { DoA } \\
\left(\mathrm{m}^{2} \mathrm{ha}^{-1}\right)\end{array}$ & $\begin{array}{l}\text { DoR } \\
(\%)\end{array}$ & $\mathbf{V C}$ & VI \\
\hline \multirow[t]{2}{*}{ Euphorbiaceae } & Jatropha mollissima (Pohl) Baill. & 0,022 & 7 & 0,85 & 0,24 & 4,29 & 0,16 & 0,01 & 0,86 & 5,15 \\
\hline & $\begin{array}{l}\text { Manihot carthaginensis (Jacq.) Müill. } \\
\text { Arg. }\end{array}$ & 0,050 & 6 & 0,73 & 0,16 & 2,86 & 0,30 & 0,02 & 0,75 & 3,61 \\
\hline Fabaceae & $\begin{array}{c}\text { Anadenanthera colubrina (Vell.) } \\
\text { Brenan }\end{array}$ & 0,022 & 5 & 0,61 & 0,12 & 2,14 & 0,11 & 0,01 & 0,61 & 2,76 \\
\hline Malvaceae & $\begin{array}{l}\text { Pseudobombax marginatum (A. St.- } \\
\text { Hil.) A. Robins }\end{array}$ & 0,016 & 4 & 0,48 & 0,12 & 2,14 & 0,06 & 0,00 & 0,49 & 2,63 \\
\hline Anacardiaceae & Spondias tuberosa Arruda & 0,202 & 3 & 0,36 & 0,12 & 2,14 & 0,61 & 0,04 & 0,41 & 2,55 \\
\hline$\dagger$ & Morto & 0,045 & 3 & 0,36 & 0,08 & 1,43 & 0,14 & 0,01 & 0,37 & 1,80 \\
\hline Cactaceae & $\begin{array}{l}\text { Pilosocereus gounellei (F. A. C. } \\
\text { Weber) Byles \& Rowley }\end{array}$ & 0,023 & 3 & 0,36 & 0,12 & 2,14 & 0,07 & 0,01 & 0,37 & 2,51 \\
\hline Euphorbiaceae & Croton blanchetianus Baill. & 0,019 & 3 & 0,36 & 0,08 & 1,43 & 0,06 & 0,00 & 0,37 & 1,80 \\
\hline Fabaceae & Bauhinia cheilantha (Bong.) Steud. & 0,012 & 3 & 0,36 & 0,12 & 2,14 & 0,04 & 0,00 & 0,37 & 2,51 \\
\hline Anacardiaceae & Schinopsis brasiliensis Engl. & 0,267 & 2 & 0,24 & 0,08 & 1,43 & 0,53 & 0,04 & 0,28 & 1,71 \\
\hline Celastraceae & Fraunhofera multiflora Mart. & 0,183 & 2 & 0,24 & 0,08 & 1,43 & 0,37 & 0,03 & 0,27 & 1,70 \\
\hline Erythroxylaceae & Erythroxylum revolutum Mart. & 0,010 & 2 & 0,24 & 0,04 & 0,71 & 0,02 & 0,00 & 0,24 & 0,96 \\
\hline Cactaceae & Pilosocereus pachycladus F. Ritter & 0,013 & 1 & 0,12 & 0,04 & 0,71 & 0,01 & 0,00 & 0,12 & 0,84 \\
\hline Capparaceae & Cynophalla hastata (Jacq.) J. Presl & 0,009 & 1 & 0,12 & 0,04 & 0,71 & 0,01 & 0,00 & 0,12 & 0,84 \\
\hline Erythroxylaceae & Erythroxylum pugens O. E. Schulz & 0,005 & 1 & 0,12 & 0,04 & 0,71 & 0,00 & 0,00 & 0,12 & 0,84 \\
\hline Fabaceae & $\begin{array}{l}\text { Senna macranthera var. pudibunda } \\
\text { (Benth.) H. S. Irwin \& Barneby }\end{array}$ & 0,004 & 1 & 0,12 & 0,04 & 0,71 & 0,00 & 0,00 & 0,12 & 0,84 \\
\hline Combretaceae & Combretum monetaria Mart. & 0,003 & 1 & 0,12 & 0,04 & 0,71 & 0,00 & 0,00 & 0,12 & 0,84 \\
\hline
\end{tabular}

Dados apresentados em ordem decrescente de valor de importância $(\mathrm{VI})$, onde: $\mathrm{AB}=$ área basal; $\mathrm{DA}=$ densidade absoluta; $\mathrm{DR}=$ densidade relativa; $\mathrm{FA}=$ frequência absoluta; $\mathrm{FR}$ = frequência relativa; $\mathrm{DoA}=$ dominância absoluta; $\mathrm{DoR}=$ dominância relativa; $\mathrm{VC}=$ valor de cobertura; $\mathrm{VI}=$ valor de importância.

Tabela 2. Estimadores de riqueza CHAO 1 e CHAO 2 em remanescente na Depressão Sertaneja Meridional, Petrolina, PE.

Table 2. Richiness estimators CHAO 1 and CHAO 2 in remnant in the Southern Country Depression, Petrolina, PE.

\begin{tabular}{cccc}
\hline & Riqueza & $\begin{array}{c}\text { S (est) } \\
\text { Limite inferior }\end{array}$ & $\begin{array}{c}\text { S (est) } \\
\text { Limite superior }\end{array}$ \\
\hline S(est) & 27 & 23,57 & 30,43 \\
Chao 1 & 29,5 & 27,37 & 43,97 \\
Chao 2 & 29,4 & 27,39 & 41,77 \\
\hline
\end{tabular}

Estimativa com intervalo de confiança de $95 \%$, onde $\mathrm{S}=$ riqueza observada.

\section{Parâmetros estruturais}

As dez espécies que apresentaram os maiores valores de importância (VI) na comunidade, em ordem decrescente, foram: Mimosa tenuiflora $(113,99)$, Poincianella microphylla $(68,5)$, Commiphora leptophloeos $(23,4)$, Cnidoscolus quercifolius $(14,1)$, Piptadenia stipulacea $(13,7)$, Croton rhamnifolius $(8,8)$, Poincianella pyramidalis $(5,6)$, Aspidosperma pyrifolium (5,5), Jatropha molissima (5,2) e Mimosa hexandra $(5,1)$ somando $87,9 \%$ do VI total (Tabela 2). Estas espécies foram encontradas em $28 \%$ ou mais das unidades amostrais, exceto Poincianella pyramidalis, que foi observada em apenas $8 \%$. Mimosa tenuiflora foi a mais importante na comunidade, sobretudo por sua grande dominância relativa $(63,2 \%)$, alta frequência $(15,0 \%)$ e alta densidade relativa $(35,8 \%)$.

A baixa densidade encontrada para os cactos conhecidos como xique-xique, Pilosocereus pachycladus ( 1 ind ha ${ }^{-1}$ ) e P.gounellei ( 3 ind ha ${ }^{-1}$ ) tem relação com a própria sobrevivência dos mesmos na área de estudo. A maioria encontrava-se morta ou perecendo durante os trabalhos de campo, não sendo possível contabilizá-las na amostragem.

Analisando as espécies consideradas intermediárias na amostragem, ou seja, não eram nem as mais importantes nem as raras, destacaram-se algumas ecofisiologicamente exigentes em luz e clímax (Gamarra-Rojas \& Sampaio, 2002; Moro et al., 2014) como é o caso de Anadenanthera 
colubrina (5), Pseudobombax marginatum (4) e Spondias tuberosa (3). Suas densidades absolutas (entre parênteses) denotam que suas populações não são numerosas e, por serem madeiras comerciais (exceto a segunda), sua conservação está ameaçada. Mesmo assim, suas presenças também corroboram com a interpretação de avanço da sucessão na área de estudo, uma vez que seus crescimentos são mais lentos e dependem de microclima mais ameno (sombreado).

Os indivíduos amostrados foram distribuídos em quatro classes de altura com intervalos crescentes (Figura 2). Na faixa de 1,5 $\mathrm{m}$ a $6 \mathrm{~m}$ de altura observou-se $98,7 \%$ das espécies inventariadas. As maiores alturas foram registradas para Schinopsis brasiliensis e Cnidoscolus quercifolius, com $8 \mathrm{~m} \mathrm{e} 7 \mathrm{~m}$ respectivamente, perfazendo $1,1 \%$ do total amostrado.

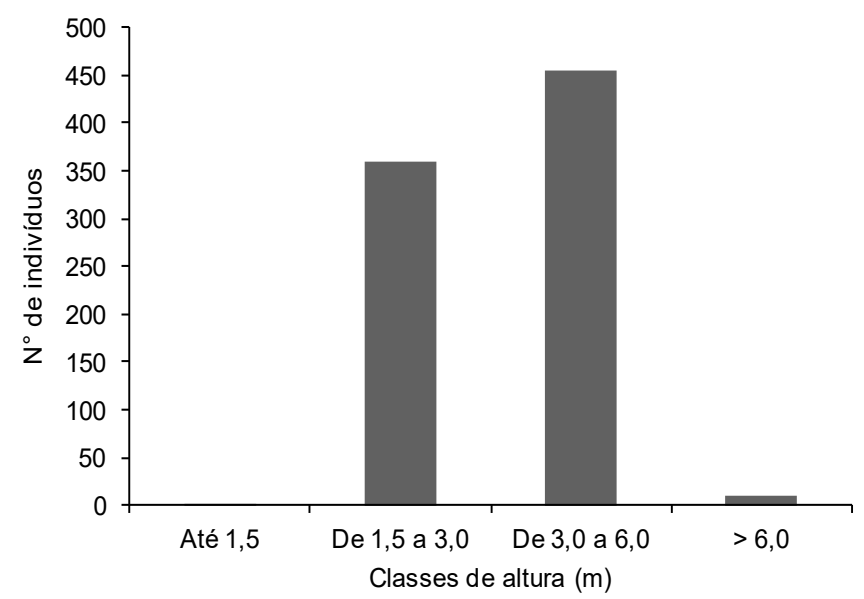

Figura 2. Distribuição em classes de altura dos indivíduos amostrados em remanescente na Depressão Sertaneja Meridional, Petrolina, PE.

Figure 2. Distribution in height classes of the individuals sampled in remnant in Southern Sertaneja Depression, Petrolina, PE.

As menores alturas foram registradas para Pilosocereus gounellei e Commiphora leptophloeos, com 0,9 m e $1,4 \mathrm{~m}$, respectivamente. Nessa classe de altura, encontrase apenas $0,24 \%$ dos indivíduos registrados na área, ressaltando que o critério de inclusão escolhido foi pertinente para amostrar uma vegetação de porte arbustivo-arbóreo.

A distribuição dos indivíduos em classes diamétricas é apresentada na Figura 3. A segunda classe diamétrica $(8-14 \mathrm{~cm})$, foi a que apresentou o maior número de indivíduos, estando presente nessa classe $45,1 \%$ do total amostrado. Na primeira classe $(5-8 \mathrm{~cm})$, concentraram-se $39,0 \%$ dos indivíduos.

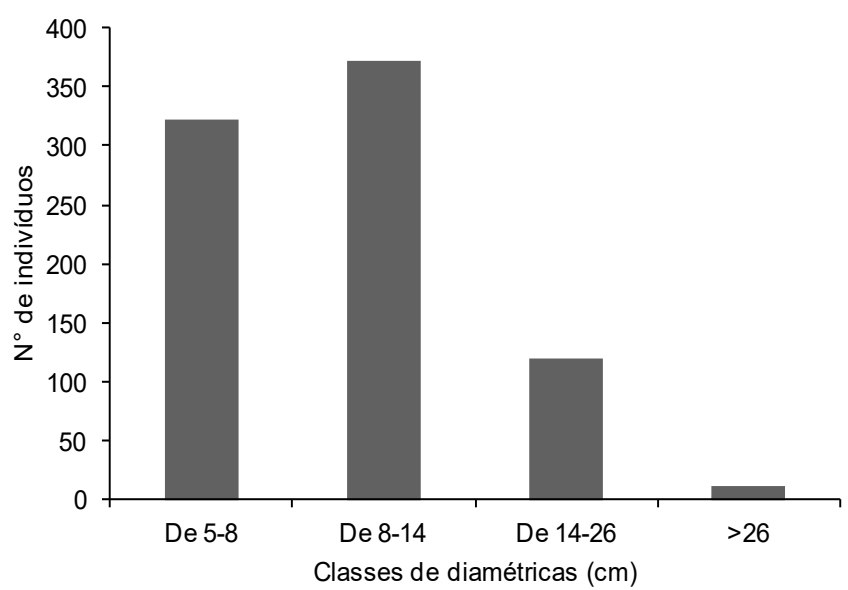

Figura 3. Distribuição da área basal em classes diamétricas dos indivíduos amostrados em remanescente na Depressão Sertaneja Meridional, Petrolina, PE.

Figure 3. Distribution of the basal area in diametric classes of individuals sampled in remnant in Southern Sertaneja Depression, Petrolina, PE.

\section{Heterogeneidade}

Dentre os modelos de distribuição de espécies testados, apenas o normal logarítmico (Figura 4) se ajustou significativamente $\left(\mathrm{X}^{2}=2,65, \mathrm{p}=0,618\right)$, com gradientes longos (Figura 5).

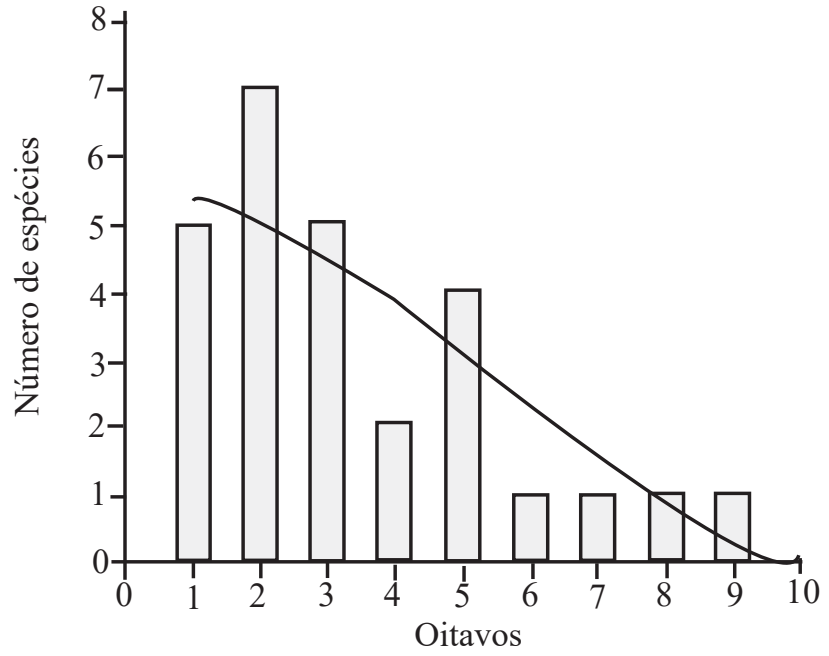

Figura 4. Modelo normal logarítmico para os dados de abundância e riqueza do componente arbustivo-arbóreo em remanescente na Depressão Sertaneja Meridional, Petrolina, PE.

Figure 4. Normal logarithmic model for data of abundance and richness of the shrub-arboreal component in remnant in Southern Sertaneja Depression, Petrolina, PE. 


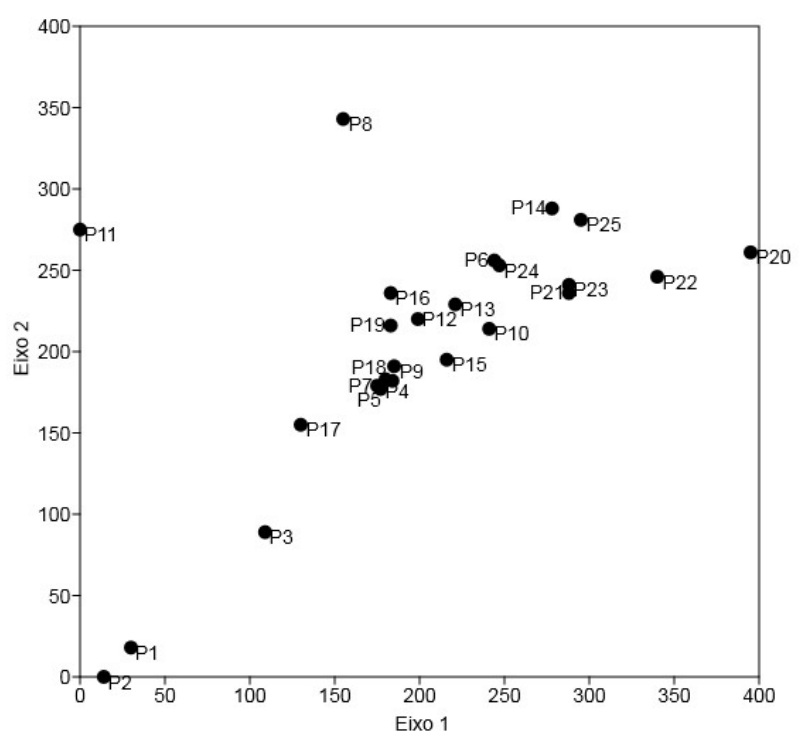

Figura 5. Análise de correspondência segmentada, mostrando a distribuição das parcelas nos eixos de ordenação, onde: Eixo $1=0,66$ e Eixo $2=0,3$.

Figure 5. Segmented correspondence analysis, showing the distribution of plots in the ordering axes, where: Axis $1=0.66$ and Axis $2=0.3$.

\section{Discussão}

A riqueza encontrada (27 espécies) foi considerada intermediária, em comparação com levantamentos na mesma ecorregião e com amostragem semelhante (Calixto Júnior \& Drumond, 2011; Barbosa et al., 2012; Fabricante et al., 2012; Ferraz et al., 2014). Por exemplo, foi maior que o encontrado por Ferraz et al. (2014) (25 espécies) e inferior ao encontrado por Barbosa et al. (2012) (36 espécies). Fabaceae e Euphorbiaceae são famílias que têm demonstrado alta riqueza em levantamentos realizados em diferentes áreas de Caatinga da Depressão Sertaneja Meridional (DSM) (Gomes et al., 2006; Moreira et al., 2007; Rodal et al., 2008a; 2008b; Calixto Júnior et al., 2009; Souza \& Rodal, 2010; Calixto Júnior \& Drumond, 2011; Barbosa et al., 2012; Fabricante et al., 2012; Pereira Júnior et al., 2012; Ferraz et al., 2014).

A área de estudo conserva uma flora indicativa de Caatinga da DSM (Tabela 2), estando de acordo com os demais levantamentos fitossociológicos dessa ecorregião (Calixto Júnior et al., 2009; Calixto Júnior \& Drumond, 2011, 2014; Barbosa et al., 2012; Fabricante et al., 2012; Ferraz et al., 2014). Os gêneros mais ricos são também os mais representativos em diversos levantamentos quantitativos na DSM (Moreira et al., 2007; Calixto Júnior et al., 2009; Fabricante et al, 2012; Pereira Júnior et al., 2012;) e, segundo Moro et al. (2014), são aqueles que caracterizam a Caatinga em avanço de sucessão no nordeste meridional. Ainda neste contexto, os gêneros Pilosocereus, Erythroxylum e Pseudobombax são chaves na manutenção dos processos ecossistêmicos, já que ou são dispersos por aves e mamíferos em razão dos frutos carnosos como os dois primeiros, ou são polinizados por morcegos e aves no caso do último. Em particular, Pseudobombax é considerado um gênero importante ecologicamente, pois floresce sempre na época de maior estiagem, fornecendo recurso alimentar à fauna polinizadora no período de maior escassez (Lima \& Rodal, 2010).

Mimosa tenuiflora é uma espécie que apresenta ampla distribuição em áreas de vegetação caducifólia espinhosa e tem sido registrada com frequência em diversos levantamentos quali-quantitativos na região do presente estudo (Calixto Júnior et al., 2009; Calixto Junior \& Drumond, 2011, 2014; Barbosa et al., 2012; Fabricante et al., 2012).

P. microphylla foi uma das espécies mais abundantes no inventário realizado em uma área de Caatinga em Petrolina, PE (Drumond et al., 2002), corroborando com o encontrado no presente estudo.

Para as espécies consideradas raras na amostragem, vale a interpretação de que suas baixas frequências são uma consequência da perturbação antrópica, uma vez que muitas dessas espécies são comumente utilizadas pela população local como forrageiras para a caprinocultura. De fato, Combretum monetaria, Cynophalla hastata e Erythroxylum pugens foram encontradas respectivamente nas parcelas 13, 17 e 22, áreas de sequeiro sem intervenção recente, onde a vegetação é mais arbórea que savânica, além do solo ser mais profundo e menos arenoso (Beltrão et al., 2005).

$\mathrm{O}$ valor de área basal $\left(8,81 \mathrm{~m}^{2} \mathrm{ha}^{-1}\right)$ foi inferior ao encontrado por Alcoforado-Filho et al. (2003) $\left(24,9 \mathrm{~m}^{2} \mathrm{ha}^{-1}\right)$ e por Santana \& Souto (2006) $\left(17,50 \mathrm{~m}^{2} \mathrm{ha}^{-1}\right)$, ambos na DSM, inferior também ao encontrado por Calixto Júnior \& Drumond (2014) em um estudo realizado em Petrolina $\left(40,23 \mathrm{~m}^{2} \mathrm{ha}^{-1}\right)$. O corte seletivo de madeira, abertura de trilhas e pastejo em parte da área estudada antes da construção do campus justifica estes valores baixos de área basal, mesmo tendo no local postos de vigilância que inibem ações de extrativismos possibilitando, mesmo que parcialmente, o avanço da sucessão. 
A espécie destacada nos resultados, por deter valores significativos de área basal (C. leptophloeos ou umburana-de-cambão), é uma árvore bastante apreciada para artesanato em função do tipo maleável e resistente de sua madeira (Silva, 2015), indicando que não vem sendo extraída de forma predatória.

A diversidade da vegetação $(\mathrm{H}\rangle)$ observada foi semelhante ao registrado em outros trabalhos realizados na DSM em Pernambuco, como relatado por Ferraz et al. (2014) (2,01 nats ind $\left.{ }^{-1}\right)$ e Barbosa et al. (2012) (2,90 nats ind $\left.{ }^{-1}\right)$. Porém, superior ao encontrado para áreas de Caatinga em Petrolina, onde os valores registrados estiveram entre 1,39 nat $\operatorname{sind}^{-1} \mathrm{e} 1,88$ nat sind $\mathrm{d}^{-1}$ (Calixto Júnior \& Drumond, 2011, 2014; Fabricante et al., 2012). Portanto, ao analisar os valores encontrados nos estudos em Petrolina, percebe-se a dominância ecológica de algumas espécies sobre as demais, em especial M. tenuiflora e $P$. microphylla, espécies também encontradas com altos valores de importância (VI) nos referidos trabalhos. É importante mencionar que a dominância ecológica moderada de algumas espécies na comunidade é um padrão comum em formações de savana estépica nas depressões sertanejas (Moro et al., 2014).

O resultado obtido para a equabilidade de Pielou foi inferior $\left(\mathrm{J}^{\prime}=0,62\right)$, quando comparado com levantamentos próximos geograficamente. Calixto Junior \& Drumond (2014) observaram J' $=0,78$, no município de Petrolina, PE e Fabricante et al. (2012) $\mathrm{J}^{\prime}=0,73$, em Juazeiro, BA, indicando menor dominância ecológica na área onde estudaram em comparação com a deste estudo.

\section{Parâmetros estruturais}

M. tenuiflora é indicadora de sucessão secundária progressiva na Caatinga, quando é praticamente a única espécie lenhosa presente, porém a tendência ao longo do processo é de redução numérica drástica com o avanço da sucessão (Araújo Filho \& Carvalho, 1996). De acordo com Bakke et al. (2006), é uma espécie de usos múltiplos e coloniza abundantemente sítios desfavoráveis, incluindo aqueles com escassez hídrica. Em seu habitat natural, tem sido explorada para produção de estacas e lenha, além de forragem para caprinos, ovinos e bovinos, que encontram nessa planta um importante componente de suas dietas (Pereira Filho et al., 2005). Portanto, é uma espécie chave no contexto de prospecção de espécies lenhosas para recuperação de áreas degradadas no bioma.
P. microphylla e C. leptophloeos também apresentaram VI mais altos, principalmente por deterem grande número de indivíduos no caso da primeira, e elevada área basal no caso da segunda (Tabela 2), refletindo na estrutura da comunidade o padrão de nicho e guilda comumente encontrado em Savanas Estépicas Arborizadas, com uma ou duas espécies nucleares (elevada área basal) e as demais com alto VI refletido de forma dispersa em populações abundantes e de pequeno porte (Moro et al., 2014). Tal estruturação comunitária pode e deve ser replicada, como método para recuperar áreas degradadas de Caatinga na região do presente estudo, sendo $P$. microphylla e C. leptophloeos também indicadas para tais ações.

Cnidoscolus quercifolius, Piptadenia stipulacea, Croton rhamnifolius, Poincianella pyramidalis e Aspidosperma pyrifolium são consideradas árvores e arbustos pioneiros nas Caatingas da DSM, sendo encontradas em grandes populações em áreas onde houve supressão total ou parcial da vegetação (Gamarra-Rojas \& Sampaio, 2002; Moro et al., 2014). Bem espalhadas na área, estas espécies auxiliam no amadurecimento da comunidade, uma vez que sua polinização é melitofilica (nas quatro primeiras) e suas dispersões são por agentes abióticos, como o vento ou a gravidade. Tal fato também corrobora com um cenário transitório, principalmente nas áreas de sequeiro, passando de estádio inicial para intermediário. Outros estudos também em formações similares registraram resultados parecidos (Guedes et al., 2012; Pereira Júnior et al., 2012).

$S$. brasiliensis é uma espécie de grande valor madeireiro, procurada para uso em construções rurais e domésticas (Ferraz et al., 2006), mas apenas dois indivíduos foram amostrados neste estudo (Tabela 2), fato que chama atenção para a conservação da mesma no remanescente em estudo. C. quercifolius é uma importante forrageira, sendo utilizada rotineiramente na suplementação alimentar de ovinos no semiárido pernambucano (Pereira et al., 2012) e sua densidade absoluta foi um pouco maior (29). Essa espécie é também produtora de pólen e néctar que atraem muitos insetos, principalmente abelhas nativas (Maia-Silva et al., 2012), sendo assim uma espécie melífera importante na Caatinga. Essas espécies são amplamente distribuídas na Caatinga, e foram registradas em outros estudos florísticos como árvores de grande porte (Guedes et al., 2012; Pereira Júnior et al., 2012). 
Para as classes de altura e diâmetro, segundo Carvalho \& Nascimento (2009), quando a distribuição dos indivíduos em classes diamétricas forma um padrão que difere do J-invertido, pode-se inferir que provavelmente em um período não muito distante, houve distúrbio na vegetação. Foi observado nesse trabalho um padrão típico de savanas tropicais (Rodal et al., 2013), onde a maioria dos indivíduos encontra-se agrupada nas classes intermediárias de altura, que variam de $1,5 \mathrm{~m}$ a 6 m. Além disso, segundo Gamarra-Rojas \& Sampaio (2002) para áreas antropizadas de savana estépica arborizada é comum que a maioria dos indivíduos se concentre em classes intermediárias de diâmetro, muito em função de alguns indivíduos serem poupados de corte para sombrear o gado. Padrão semelhante ao encontrado nesse estudo foi descrito por Calixto Junior \& Drumond (2014), no estudo comparativo da estrutura fitossociológica de dois fragmentos de Caatinga em Petrolina. Nesse estudo, os autores encontraram maior número de indivíduos ocorrendo entre a segunda e quarta classes, considerando que as classes foram as mesmas em ambos estudos.

O padrão encontrado difere do J-invertido, que tem sido observado com frequência nos estudos realizados na Caatinga local (Calixto Junior \& Drumond, 2011; Fabricante et al., 2012; Guedes et al., 2012). A concentração de indivíduos nas menores classes diamétricas, fazendo com que o padrão se aproxime do J-invertido, é comum e esperado, principalmente em ecossistemas onde está ocorrendo sucessão. Isso indica o balanço positivo entre recrutamento e mortalidade, caracterizando a comunidade como autorregenerante (Silva Júnior, 2004). Porém, como mencionado, é possível que algumas árvores de interesse tenham sido conservadas por proprietários anteriores da área de estudo, como é o caso da umburana-de-cambão ( $C$. leptophloeos) e da baraúna (S. brasiliensis).

\section{Heterogeneidade}

O ajuste ao modelo normal logarítmico foi um resultado já esperado, pois este é o modelo que normalmente se ajusta a comunidades tropicais heterogêneas. Tal heterogeneidade é corroborada na análise de ordenação (DCA), que apresentou elevados autovalores nos dois primeiros eixos canônicos $(0,66$ para o eixo 1 e 0,3 para o eixo 2), valores tidos como significativos $(>0,30)$ em estudos de gradientes em comunidades tropicais heterogêneas (Felfili et al., 2005), suportando a elevada heterogeneidade florística na comunidade e evidenciando uma maior diferenciação em termos de repartição de recursos.

Mesmo com a dominância das espécies Mimosa tenuiflora (jurema-preta) e Poincianella microphylla (catingueira), os demais modelos não se ajustaram, mostrando haver grande heterogeneidade na repartição dos recursos (ocupação de nichos) na comunidade (Magurran, 1988). De fato, em outros levantamentos na região a dominância ecológica das espécies mais importantes não interferiu na heterogeneidade da comunidade quando avaliada (Fabricante et al., 2012; Guedes et al., 2012). A carência de análises desta natureza em levantamentos quantitativos no bioma Caatinga restringe bastante sua discussão.

\section{Conclusões}

A vegetação de Caatinga remanescente nas Depressões Sertanejas encontra-se bastante fragmentada e empobrecida. Em regiões onde há forte vocação agrícola este cenário é ainda pior. No Vale do São Francisco as reservas legais em Fazendas e demais áreas de uso púbico ganham enorme importância no contexto conservacionista. Os resultados aqui apresentados servirão como base para futuros estudos de dinâmica da vegetação e a continuidade dos estudos no local por essa equipe de autores garantirá o subsídio e a argumentação necessários para a implementação das ações de conservação. Nesse contexto, os remanescentes de caatinga da DSM na região do vale do São Francisco começam a ter sua flora arbórea conhecida, passo primordial para que seja, de fato, conservada.

\section{Referências}

Alcoforado-Filho, F. G. et al. Florística e fitossociologia de um remanescente de vegetação caducifólia espinhosa arbórea em Caruaru, Pernambuco. Acta Botanica Brasilica, v. 17, n. 2, p. $287-$ 303, 2003. DOI: 10.1590/S0102-33062003000200011.

Alves, J. J. A. et al. Degradação da Caatinga: uma investigação ecogeográfica. Revista Caatinga, v. 22, n. 3, p. 126-135, 2009.

Alves, J. J. A. Geoecologia da Caatinga no Semiárido do Nordeste. CLIMEP: Climatologia e Estudos da Paisagem, v. 2, n. 1, p. 58-71, 2007.

The Angiosperm Phylogeny Group. An update of the Angiosperm Phylogeny Group. Classification for the orders and families of flowering plants: APG IV. Botanical Journal of the Linnean Society, v. 181, p. 1-20, 2016. DOI: 10.1111/boj.12385. 
Araújo Filho, J. A. \& Carvalho, F. C. Desenvolvimento sustentado da Caatinga. In: Alvarez, V. H.; Fontes, L. E. F.; Fontes, M. P. O solo nos grandes domínios morfoclimáticos do Brasil e o desenvolvimento sustentado. Viçosa, MG: SBCS, UFV, DPS, 1996.

Araújo Filho, J. C. et al. Levantamento de reconhecimento de baixa e média intensidade dos solos do Estado de Pernambuco. Rio de Janeiro: Embrapa Solos, 2000. 378 p.

Bakke, I. A. et al. Water and sodium chloride effects on Mimosa tenuiflora (Willd.) Poiret seed germination. Revista Caatinga, v. 19, n. 3, p. 261-267, 2006.

Barbosa, M. D. et al. Florística e fitossociologia de espécies arbóreas e arbustivas em uma área de Caatinga em Arcoverde, PE, Brasil. Revista Árvore, v. 36, n. 5, p. 851-858, 2012. DOI: 10.1590/S010067622012000500007.

Begon, M. et al. Ecology: individuals, populations and communities. Malden: Blackwell Science, 1996. 738 p.

Beltrão, B. A. et al. Projeto de cadastro de fontes de abastecimento por água subterrânea: relatório diagnóstico do munícipio de Petrolina. Recife: Companhia de Pesquisa de Recursos Minerais, 2005.

Brummit, R. K. \& Powell, C. E. Authors of plant names. Kew: Royal Botanic Gardens, 1992.

Brasil. Ministério do Meio Ambiente. Biodiversidade brasileira: avaliação e identificação de áreas prioritárias para conservação, utilização sustentável e repartição de benefícios da biodiversidade brasileira. Brasília, DF, 2002.

Brasil. Ministério do Meio Ambiente. Caderno da região hidrográfica do São Francisco. Brasilia, DF, 2006.

Calixto Junior, J. T. \& Drumond, M. A. Estrutura fitossociológica de um fragmento de Caatinga sensu stricto 30 anos após corte raso, Petrolina-PE, Brasil. Revista Caatinga, v. 24, n. 2, p. 67-74, 2011.

Calixto Junior, J. T. \& Drumond, M. A. Estudo comparativo da estrutura fitossociológica de dois fragmentos de Caatinga em níveis diferentes de conservação. Pesquisa Florestal Brasileira, v. 34, n. 80, 2014. DOI: 10.4336/2014.pfb.34.80.670.

Calixto Júnior, J. T. et al. Estrutura fitossociológica de um fragmento de Caatinga sensu stricto no sertão de Pernambuco. In: CONGRESSO NORDESTINO DE ENGENHARIA FLORESTAL, 2., 2009, Campina Grande. Evolução e perspectivas da engenharia florestal no Nordeste. Campina Grande: UFCG, 2009.

Carvalho, F. A. \& Nascimento, M. T. Estrutura diamétrica da comunidade e das principais populações arbóreas de um remanescente de Floresta Atlântica Submontana (Silva Jardim-RJ, Brasil). Revista Árvore, v. 33, p. 327-337, 2009. DOI: 10.1590/ S0100-67622009000200014.

Centro de Gestão e Estudos Estratégicos. Desertificação, degradação da terra e secas no Brasil. Brasília, DF, 2016.

Colwell, R. K. et al. Modelos e estimadores que vinculam a rarefação, extrapolação e comparação de assembleias baseadas em indivíduos e baseadas em amostras. Journal of Plant Ecology, v. 5, p. 3-21, 2012.

Drumond, M. A. et al. Inventário e sociabilidade de espécies arbóreas e arbustivas da Caatinga na região de Petrolina, PE. Brasil Florestal, v. 74, p. 37-43, 2002.
Fabricante, J. R. et al. Divergências na composição e na estrutura do componente arbustivo-arbóreo entre duas áreas de Caatinga na região do Submédio São Francisco (Petrolina, PE/Juazeiro, BA). Biotemas. v. 25, n. 3, p. 97-109, 2012. DOI: 10.5007/2175-7925.2012v25n3p97.

Felfili, J. M. et al. Análises multivariadas em estudos de vegetação. Brasília, DF: Universidade de Brasília, Departamento de Engenharia Florestal, 2005. 54 p.

Ferraz, J. S. F. et al. Estrutura do componente arbustivo-arbóreo da vegetação em duas áreas de Caatinga, no município de Floresta, Pernambuco. Revista Árvore, v. 38, n. 6, p. 1055-1064, 2014. DOI: 10.1590/S0100-67622014000600010.

Ferraz, J. S. F. et al. Valor de uso e estrutra da vegetação lenhosa às margens do riacho do Navio, Floresta, PE, Brasil. Acta Botanica Brasilica, v. 20, n. 1, p. 125-134, 2006. DOI: 10.1590/S010233062006000100012 .

Flora do Brasil. Rio de Janeiro: Jardim Botânico do Rio de Janeiro, [2017]. Disponivel em: <http://floradobrasil.jbrj.gov.br/reflora/ floradobrasil/ > . Acesso em: 1 maio 2017.

Gamarra-Rojas, C.F.L. \& Sampaio, E. V. S. B. Espécies da caatinga no banco de dados do CNIP. In: Sampaio, V.C.B. et al. (Ed.).Vegetação \& flora da Caatinga. Recife: Associação Plantas do Nordeste, 2002.

Gomes, A. P. S. et al. Florística e fitogeografia da vegetação arbustiva subcaducifólia da Chapada de São José, Buíque, PE, Brasil. Acta Botanica Brasilica, v. 20, n. 1, p. 37-48, 2006. DOI: 10.1590/S010233062006000100005.

Guedes, R. S. et al.Caracterização florítica-fitossociológica do componente lenhoso de um trecho de Caatinga no semiárido paraibano. Revista Caatinga, v. 25, n. 2, p. 99-108, 2012.

Hammer, Ø. et al. PAST: paleontological statistics, version 1.81: manual. Oslo: Natural History Museum, [2008]. Avaliable from: $<$ http://folk.uio.no/ohammer/past>. Access on: 6 May 2018.

IBGE. Manual técnico da vegetação brasileira. 2. ed. Rio de Janeiro, 2012. $271 \mathrm{p}$.

Lima, A. L. A. \& Rodal, M. J. N. Phenology and wood density of plants growing in the semi-arid region of northeastern Brazil. Journal of Arid Environments, v. 74, n. 11, p. 1363-1373, 2010. DOI: 10.1016/j.jaridenv.2010.05.009.

Maia-Silva, C. et al. Guia de plantas visitadas por abelhas na Caatinga. Fortaleza: Fundação Brasil Cidadão, 2012.

Magurran, E. A. Ecological diversity and its measurement. 2. ed. Princeton: Princeton University Press, 1988. 179 p.

Martinelli, G. \& Moraes, M. A. (Ed). Livro vermelho da flora brasileira. Rio de Janeiro: Centro Nacional de Conservação da Flora, Instituto de Pesquisas Jardim Botânico do Rio de Janeiro, 2013. 1100 p.

Martins, F. R. Estrutura de uma floresta mesófila. Campinas: Ed. Unicamp, 1991.

Moreira, A. R. P. et al. Composição florística e análise fitossociológica arbustivo-arbóreo no município de Caraúbas-RN. Revista Verde, v. 2, n. 1, p. 113-126, 2007. 
Moro, M. F. et al. A catalogue of the vascular plants of the Caatinga Phytogeographical Domain: a synthesis of floristic and phytosociological surveys. Phytotaxa, v. 160, n. 1, p. 118, 2014. DOI: 10.11646/phytotaxa.160.1.1.

Mueller-Dombois, D. \& Ellenberg, H. Aims and methods of vegetation ecology. New York: John Wiley \& Sons, 1974.

Ortega, A. C. \& Sobel, T. F. Desenvolvimento territorial e perímetros irrigados: avaliação das políticas governamentais implantadas nos Perímetros Irrigados Bebedouro e Nilo Coelho em Petrolina (PE). Planejamento e Políticas Públicas, n. 35, p. 87-118, 2010.

Pereira Filho, J. M. et al. Correlação entre o teor de tanino e a degradabilidade ruminal da matéria seca e proteína bruta do feno de jurema-preta (Mimosa tenuiflora Wild) tratada com hidróxido de sódio. Livestok Research of Rural Development,v. 17, n. 8, 2005.

Pereira Júnior, L. R. et al. Composição florística e fitossociológica de um fragmento de Caatinga em Monteiro, PB. HOLOS, v. 6, p. 73-87, 2012.

Pereira, V. L. A. et al. Valor nutritivo e consumo voluntário do feno de faveleira fornecido a ovinos no semiárido pernambucano. Revista Caatinga. v. 25, n. 3, p. 96-101, 2012.

Rodal, M. J. N. et al. Estrutura da vegetação caducifólia espinhosa (Caatinga) de uma área do sertão central de Pernambuco. Hoehnea, v. 35, n. 2, p. 209-217, 2008 a.

Rodal, M. J. N. et al. Levantamento quantitativo da plantas lenhosas em trechos de vegetação de Caatinga em Pernambuco. Revista Caatinga. v. 21 , n. 3, p. 192-205, 2008b. DOI: 10.1590/S223689062008000200004 .
Rodal, M. J. N. et al. Manual sobre métodos de estudos florísticos e fitossociológicos: ecossistema Caatinga. Brasília, DF: Sociedade Botânica do Brasil, 2013.

Santana, J. A. D. S. \& Souto, J. S. Diversidade e estrutura fitossociológica da Caatinga na Estação Ecológica do Seridó - RN. Biologia e Ciências da Terra,v. 6, n. 2, 2006.

Schilling, A. C. \& Batista, J. L. F. Curva de acumulação de espécies e suficiência amostral em florestas tropicais. Revista Brasileira de Botânica, v. 31, n. 1, p. 179-187, 2008. DOI: 10.1590/S01004042008000100016 .

Silva Júnior, M. C. Fitossociologia e estrutura diamétrica da mata de galeria do Taquara, na reserva ecológica do IBGE, DF. Revista Árvore, v. 28, n. 3, p. 419-428, 2004. DOI: 10.1590/S010067622004000300013 .

Silva, R. C. S. Estudo fitossociológico de Commiphora leptophloeos (Mart.) J. B. Gillet. e análise socioeconômica de sua utilização na atividade artesanal em Ibimirim, PE. 2015. 67 f. Dissertação (Mestrado em Ciências Florestais) - Universidade Federal Rural de Pernambuco, Recife.

Souza, J. A. N. \& Rodal, M. J. N. Levantamento florístico em trecho de vegetação ripária de Caatinga no Rio Pajeú, Floresta/PernambucoBrasil. Revista Caatinga, v. 23, n. 4, p. 54-62, 2010.

Souza, K. et al. Fauna de lagartos de hábitats de Caatinga do Campus de Ciências Agrárias da Universidade Federal do Vale do São Francisco. Revista Brasileira de Zoociências, v. 15, n. 1,2,3, p. $253-265,2013$. 